\title{
Post-Traumatic Subfrontal with Orbital Extradural Hematoma and Proptosis (Unusual Presentation Managed with Needle Aspiration): A Case Report with Review of Literature
}

\author{
Sumit Raj ${ }^{1}$ Pradeep Chouksey ${ }^{1}$ Adesh Shrivastava ${ }^{1}$ Rakesh Mishra ${ }^{1}$ Manas Prakash ${ }^{1}$ Amit Agrawal ${ }^{1}$ \\ ${ }^{1}$ Department of Neurosurgery, All India Institute of Medical \\ Sciences, Bhopal, Madhya Pradesh, India

\begin{abstract}
Address for correspondence Sumit Raj, MCh, Department of Neurosurgery, All India Institute of Medical Sciences, Saket Nagar, Bhopal, Madhya Pradesh 462001, India

(e-mail: dr.sumitraj.neurosurgery@gmail.com).
\end{abstract}

Indian J Neurotrauma 2022;19:49-51.

\begin{abstract}
Keywords

- proptosis

- needle aspiration

- orbital extradural hematoma

- subfrontal extradural hematoma

- traumatic extradural hematoma

Extradural hematoma (EDH) is a common intracranial pathology following motor vehicle accidents, comprising approximately 0.2 to $6 \%$ of all head injuries. The association of EDH with subperiosteal intraorbital hematomas is rarely reported. We report a case of a traumatic subfrontal EDH with subperiosteal hematoma (orbital EDH) successfully treated with needle aspiration, demonstrating that in appropriate patients, needle aspiration can result in the resolution of symptoms without an invasive procedure.
\end{abstract}

\section{Introduction}

Extradural hematoma (EDH) is a common intracranial pathology following motor vehicle accidents, comprising approximately 0.2 to $6 \%$ of all head injuries. ${ }^{1}$ The higher incidence is found in patients with more severe injuries. The association of EDH with subperiosteal intraorbital hematomas is rarely reported. ${ }^{2}$ Patients usually present with signs of increased intracranial pressure with loss of consciousness after a lucid interval. However, the diagnosis of frontal EDH can be missed or delayed due to minimal neurological sign which would not require a computed tomography of brain during acute presentation. Proptosis is a rare presentation of frontal EDH unless it is associated with subperiosteal extension into the orbital cavity. ${ }^{2,3}$ Delay in the treatment of this condition may lead to permanent blindness. Treatment options include observation, needle aspiration, and surgical evacuation. ${ }^{3}$ We report a case of a traumatic subfrontal EDH with subperiosteal hematoma (orbital EDH) successfully treated with needle aspiration, demonstrating that in appropriate patients, needle aspiration can result in resolution of symptoms without a more invasive procedure.

\section{Case}

A 13 years old male child presented in the emergency department with proptosis and chemosis of the left eye and inability to close the left eyelid for 6 to 7 days. The patient had a history of RTA around 11 days back followed by transient loss of consciousness. At the time of injury, the vision was unaffected. The patient consulted a local doctor and was published online

June 1, 2021
DOI https://doi.org/

$10.1055 / \mathrm{s}-0041-1725572$ ISSN 0973-0508. (c) 2021. Neurotrauma Society of India.

This is an open access article published by Thieme under the terms of the Creative Commons Attribution-NonDerivative-NonCommercial-License, permitting copying and reproduction so long as the original work is given appropriate credit. Contents may not be used for commercial purposes, or adapted, remixed, transformed or built upon. (https://creativecommons.org/licenses/by-nc-nd/4.0/).

Thieme Medical and Scientific Publishers Pvt. Ltd. A-12, 2nd Floor, Sector 2, Noida-201301 UP, India 
discharged on analgesics. After 3 days patient complained of swelling of the left eye along with tearing from the left eye. Within 2 days, the swelling increased and the vision started deteriorating for which the patient consulted in a hospital.

CT brain was done which was suggesting an extradural collection in the left frontal region along with which a retro-orbital collection in the left eye (-Fig. 2A and 2B). USG orbit (B Scan) was done: approx. $15 \mathrm{ml}$ collection with thick echogenic content seen above the left eyeball suggestive of

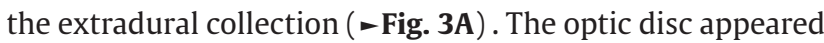
normal and chorio- retinal complex appears normal. The eye lens was normal. There are few thin echogenic membranes seen in the vitreous cavity.

The patient was referred to a higher center from the local hospital but did not consult anywhere till vision deteriorated further. On the eleventh day, the patient was brought to the emergency department of our institute. On presentation, the patient was conscious and oriented. He had proptosis of the left eye with corneal ulceration and epiphora (-Fig. 1A). Visual acuity of the right eye was 6/6 and that of the left eye was the only perception of light (PL +ve).

Repeat CT Brain was suggestive of well-defined extra-axial hypo dense (+20 HU) collection of approx. size $4.5 \times 2.6 \times 20$ $\mathrm{mm}$ (AP X TR x CE) noted in the left basi-frontal region seen compressing the adjacent brain parenchyma, however, no significant midline shift noted. Well-defined hypodense collection (+22 HU) of approx. size $3.4 \times 2.9 \times 1.7 \mathrm{~mm}$ (AP x TR X $\mathrm{CE}$ ) noted in the superior wall of left orbit seen abutting the superior rectus and displacing the eyeball forward outward and inferiorly. No obvious bony fracture was noted.

Ophthalmology consultation was done and the patient was admitted to the Neurosurgery department. After informed consent, the retro-orbital collection was aspirated under USG guidance. About $15 \mathrm{ml}$ of dark brown color (hemorrhagic) fluid was aspirated ( - Fig. 3B). The proptosis was decreased and with a significant reduction in chemosis in the next few days ( - Fig. 1B) with eye patching of the left eye. After 5 days, a repeat CT brain showed a significant decrease in the left frontal extradural collection with some residual retro-orbital collection. The residual collection was re-aspirated (about $8 \mathrm{ml}$ ) under USG guidance. CT brain after re-aspiration revealed almost near to complete resolution of the frontal and the retroorbital collection ( - Fig. 4A and 4B). Finally, the patient was transferred to the ophthalmology department for management of the corneal ulcer.

\section{Discussion}

Proptosis is an uncommon presentation in an EDH of the frontal area. This happen due to subperiosteal extension of hematoma into the orbital cavity. ${ }^{1,2}$ Proptosis can be the sole finding in these cases. ${ }^{2}$ This extension is frequently unilateral but can be bilateral. ${ }^{2}$ Proptosis and exophthalmos can present directly after the wound or have delayed presentation. ${ }^{1,4,5}$

There are several cases of post-traumatic subperiosteal hematoma reported in the literature. ${ }^{1,2,4,6-14}$ This uncommon entity is usually associated with the fracture of orbital roof. ${ }^{1}$ A subperiosteal hematoma usually presents with painful

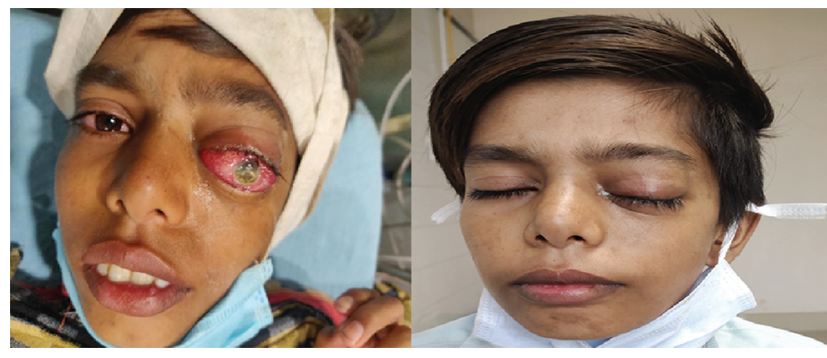

Fig. 1 (A) Preaspiration picture showing proptosis, conjunctival chemosis, and corneal opacity of left eye. (B) Postaspiration picture showing complete eye closure and resolution of proptosis.

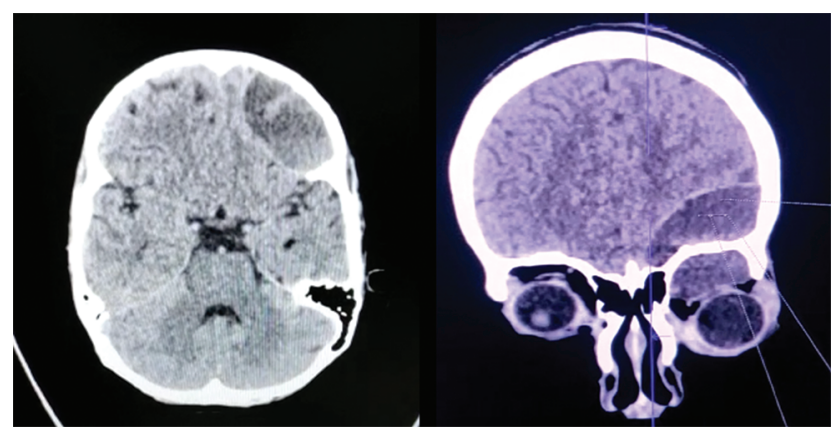

Fig. 2 Preoperative noncontrast computed tomography of the brain, (A) axial cut, and (B) coronal cut showing left frontal and retro-orbital hypodense extradural hematoma.

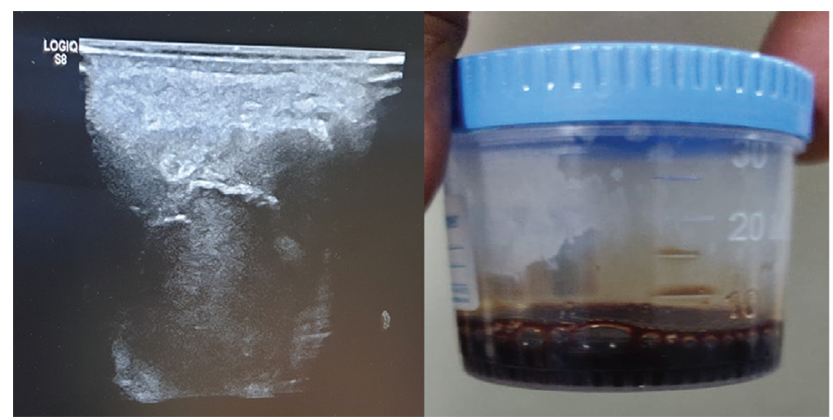

Fig. 3 Showing (A) ultrasound image and (B) image of the fluid aspirated using needle.

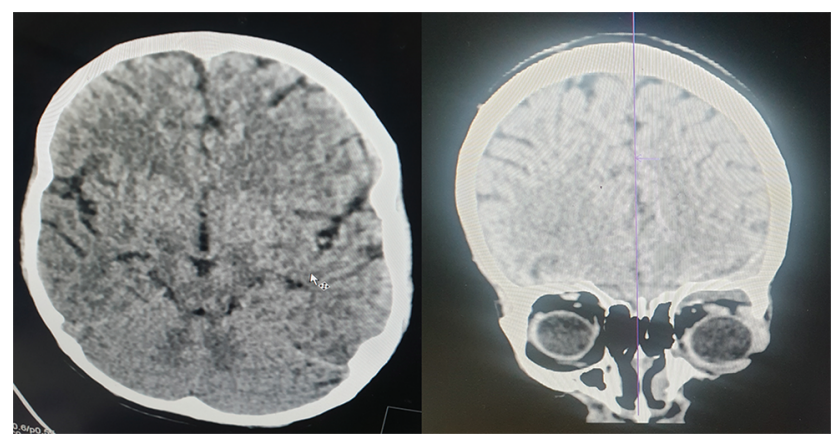

Fig. 4 Postoperative computed tomography of the brain plain, (A) axial cut, and (B) coronal cut showing complete resolution of Left frontal and orbital extradural hematoma.

proptosis and rarely with visual loss. However, if left untreated, it can progress rapidly visual loss due to retinal dysfunction, direct compression over optic nerve, and/or retinal artery 
ischemia. ${ }^{2,6,7}$ Subperiosteal extension of hematoma is seen usually just beneath the roof of the orbit. ${ }^{2,6}$ This occurs due to laceration of the subperiosteal vessels or extension of hematoma from subgaleal or subdural space. ${ }^{4,6,7,10}$ It usually occurs in young children and adolescent age group..$^{1,2,6,7}$ Since the subperiosteal extension of hematoma can be delayed for months, it is imperative to consider this diagnosis in any adolescent with post-traumatic proptosis, vertical diplopia, and visual dysfunction. ${ }^{4,15}$

Magnetic resonance imaging and computed tomography imaging are very useful for diagnosis of an intraorbital subperiosteal hematoma. ${ }^{1,6,15}$ These imaging techniques, particularly $\mathrm{CT}$, also help in identifying associated fractures. Angiography findings in these cases usually consist of stretching and inferior displacement of ophthalmic artery. ${ }^{4}$

Management depends on EDH volume, neurological and ocular findings. Large EDH needs surgical evacuation. Small size EDH with small intraorbital extension can be managed medically with close observation if the patient does not have any neurological deficits or ocular findings. However, a hematoma is likely to clear over a longer time in patients medically managed because orbital subperiosteal compartment is an avascular space. Patients with ocular findings need surgical management with frontal craniotomy, orbitotomy, and evacuation of EDH. ${ }^{1,2,5,8}$ Though needle aspiration of intraorbital hematoma is described as one of the management options, surgical management offers better and comprehensive treatment options for this unusual entity. ${ }^{6,10}$ Although technically challenging yet needle aspiration of hematoma is associated with an increased risk of rebleeding and incomplete hematoma clearance. ${ }^{9}$ Untimely and delayed management can lead to visual loss, strabismus, and permanent exophthalmos. ${ }^{2,6}$

\section{Conclusion}

Orbital EDH with proptosis is an uncommon manifestation of traumatic brain injury. Timely management is crucial for the preservation of vision in these patients. As many patients require craniotomy for the evacuation of $\mathrm{EDH}$, we present a case wherein needle aspiration resulted in complete resolution of proptosis and EDH. With this case report, we wish to emphasize the importance of needle aspiration in management of orbital EDH as an important tool in the armamentarium of neurosurgeons.

\section{Funding}

None.

\section{Conflict of Interest}

None declared.

\section{References}

1 Saiful MN, Azmi A, Saffari MH. Proptosis presenting as a delayed sign of frontal extradural haematoma. Med J Malaysia 2007;62(2):156-157

2 da Costa LB Jr, de Andrade A, Henriques JG, Cordeiro AF, Maciel CdoJ. Traumatic bilateral intraorbital (subperiosteal) hematoma associated with epidural hematoma: case report. Arq Neuropsiquiatr 2003;61(4):1039-1041

3 Rojas MC, Eliason JA, Fredrick DR. Needle aspiration of a traumatic subperiosteal haematoma of the orbit. Br J Ophthalmol 2002;86(5):593-594

4 Seigel RS, Williams AG, Hutchison JW, Wolter JR, Carlow TJ, Rogers DE. Subperiosteal hematomas of the orbit: angiographic and computed tomographic diagnosis. Radiology 1982; 143(3):711-714

5 Iwata A, Matsumoto T, Mase M, Yamada K. Chronic, traumatic intraconal hematic cyst of the orbit removed through the fronto-orbital approach: case report. Neurol Med Chir (Tokyo) 2000;40(2):106-109

6 Sharma AK, Diyora BD, Shah SG, et al. Orbital subperiosteal hematoma associated with subfrontal extradural hematoma. J Trauma 2007;62(2):523-525

7 O'Neill OR, Delashaw JB, Phillips JP. Subperiosteal hematoma of the orbit associated with subfrontal extradural hematoma: case report. Surg Neurol 1994;42(4):308-311

8 Bourdon EL, Riffaud L, Godey B, Morandi X. [Subperiosteal hematoma of the orbit associated with a frontal extradural hematoma]. J Fr Ophtalmol 1999;22(6):659-661

9 Naja A, Chellaoui A, Ibahioin K, et al. [Subperiosteal hematoma of the orbit associated with subfrontal extradural hematoma]. Neurochirurgie 2002;48(2-3 Pt 1):101-103

10 Stewart CR, Salmon JF, Domingo Z, Murray AD. Proptosis as a presenting sign of extradural haematoma. $\mathrm{Br} \mathrm{J}$ Ophthalmol 1993;77(3):179-180

11 Landa MS, Landa EH, Levine MR. Subperiosteal hematoma of the orbit: case presentation. Ophthal Plast Reconstr Surg 1998;14(3):189-192

12 Umansky F, Pomeranz S. Epidural haematoma and unilateral exophthalmos-a review. Acta Neurochir (Wien) 1989; 99(3-4):145-147

13 Chaves JB, Batista MS, Piske RL, Cunha KdeA, Almeida KJ. [Subperiosteal hematoma of the orbit: case report]. Arq Bras Oftalmol 2007;70(4):693-697

14 Procianoy F, Brandão Filho $\mathrm{M}$, Cruz AA, Alencar VM. Subperiosteal hematoma and orbital compression syndrome following minor frontal trauma in sickle cell anemia: case report. Arq Bras Oftalmol 2008;71(2):262-264

15 Brucoli M, Stecco A, Iaquinta C, Carriero A, Benech A. Diagnosis and treatment of orbit posttraumatic subperiosteal hemorrhage in a child, associated with a subdural intracranial hemorrhage. J Craniofac Surg 2005;16(3):407-410 